\title{
Memory State Feedback RMPC for Multiple Time-Delayed Uncertain Linear Systems with Input Constraints
}

\author{
Wei-Wei Qin, ${ }^{1}$ Gang Liu, ${ }^{1}$ Li-Xin Wang, ${ }^{1}$ and Zhi-Qiang Zheng ${ }^{2}$ \\ ${ }^{1}$ Department of Automatic Control, Xi'an Research Institute of High-Tech, Xian 710025, China \\ ${ }^{2}$ College of Mechatronics and Automation, National University of Defense Technology, Changsha 410073, China \\ Correspondence should be addressed to Wei-Wei Qin; qww_1982@163.com
}

Received 11 December 2013; Revised 1 March 2014; Accepted 13 March 2014; Published 13 April 2014

Academic Editor: Shuhui Bi

Copyright (c) 2014 Wei-Wei Qin et al. This is an open access article distributed under the Creative Commons Attribution License, which permits unrestricted use, distribution, and reproduction in any medium, provided the original work is properly cited.

\begin{abstract}
This paper focuses on the problem of asymptotic stabilization for a class of discrete-time multiple time-delayed uncertain linear systems with input constraints. Then, based on the predictive control principle of receding horizon optimization, a delayed state dependent quadratic function is considered for incorporating MPC problem formulation. By developing a memory state feedback controller, the information of the delayed plant states can be taken into full consideration. The MPC problem is formulated to minimize the upper bound of infinite horizon cost that satisfies the sufficient conditions. Then, based on the Lyapunov-Krasovskii function, a delay-dependent sufficient condition in terms of linear matrix inequality (LMI) can be derived to design a robust MPC algorithm. Finally, the digital simulation results prove availability of the proposed method.
\end{abstract}

\section{Introduction}

Model predictive control (MPC), also known as receding horizon control, has received much attention in control societies. The reason for its success is its ability to handle both constraints and time-varying behaviors [1-3]. Moreover, it has been recognized as a successful control strategy in industrial fields, especially in chemical process control such as petrochemical, pulp, and paper control. The basic concept of MPC is to solve an open-loop constrained optimization problem at each time instant and implement only the first control move of the solution. This procedure is repeated at the next time instant.

All physical systems (biological, economical, industrial, etc.) inherently possess time delay, which is often a source of poor performance and even instability. Since the parameters of uncertainties and time-delays are frequently the main cause of performance degradation and instability, there has been an increasing interest in the robust control of uncertain time-delay systems in the control literature [4]. Recently, many researchers have focused on the robust MPC of timedelay systems but only a few MPC algorithms have been developed to explicitly handle time-delayed systems [4-15].
In [4], after designing the novel robust constrained MPC for uncertain systems, the authors argued that the control scheme can be extended to a delayed system in a straightforward manner. Kwon et al. [5] suggested the simple receding horizon control for continuous time systems with state delay, where the reduction technique was adopted so that an optimal problem for state-delayed systems could be transformed into an optimal problem for delay-free ordinary systems. The suggested control method cannot guarantee the stability. Furthermore, an MPC algorithm for uncertain time-varying systems with input constraints and state delay was proposed. The uncertainty was assumed polytopic and the delay was assumed unknown but with a known upper bound in [7]. They then proved feasibilities and closed loop stabilities using the equivalence property. However, the results are rather conservative because feedback consists of only current state. A new memory state feedback was proposed for uncertain state-delayed systems with timedelay state and input constraints [11, 13]. The proposed method was derived from the minimization problem of infinite horizon cost by adopting memory state feedback with a delay index minimizing state estimation errors. But in practice there exist multiple time-delay states sometimes [14]. 
However, only a few MPC algorithms have been published that handle multiple time-delayed systems explicitly; a robust model predictive controller (RMPC) was proposed for a class of uncertain multiple state and input time-delay systems with nonlinear disturbance [14]. But the results were also conservative because feedback consists of only current state. A robust predictive control algorithm based on memory state feedback is proposed for a class of uncertain discretetime systems with input constraint and multiple state delays [15]. But the results were also conservative because feedback controller consists of only current state and a single timedelayed state.

In this section, we propose a new RMPC algorithm for multiple time-delayed uncertain linear systems with input constraints, in which the gain matrices of the memory state feedback controller consisting of only current state and all the time-delayed states are determined from the sufficient condition for cost monotonicity. The sufficient condition is derived using the delayed state dependent quadratic function. Compared with previous results [4], the min-max problem of the cost upper bound is formulated using the difference of the delayed state dependent quadratic function. Then the desired control input is obtained by solving the minimization problem, which is expressed in terms of LMIs. The proposed method allows an improvement in system performance in the sense of minimizing the upper bound of the infinite horizon cost.

The paper is organized as follows. In Section 2, the problem to be tackled is stated and some standard assumptions are introduced. Section 3 supplies a memory robust state feedback controller which is proposed for multiple time-delayed uncertain linear systems with input constraints. Section 4 minimizes the upper bound of infinite horizon cost that satisfies the sufficient conditions, in terms of linear matrix inequality (LMI). Section 5 illustrates the performance of the proposed controller through an example. Finally, in Section 6, we make some concluding remarks.

\section{Problem Statement}

We consider a class of discrete-time uncertain linear systems with multiple time-delayed states described as follows:

$$
\begin{gathered}
x(k+1)=(A+\Delta A) x(k) \\
+\sum_{\tau=1}^{l} A_{d \tau} x\left(k-d_{\tau}\right)+(B+\Delta B) u(k), \\
x(k)=\phi(k) \quad-d^{*} \leq k \leq 0, \\
-u_{\max } \leq u(k) \leq u_{\max }, \quad u_{\max }>0, \forall k \in[0, \infty),
\end{gathered}
$$

where $x(k) \in \mathbb{R}^{n}$ is the state, $u(k) \in \mathbb{R}^{m}$ is the control input, and $\phi(k) \in \mathbb{R}^{n}$ is the initial condition. $d_{\tau}>0(\tau=1, \ldots, l)$ are constant, representing the delay index of the state, and being assuming $0 \leq d_{1} \leq d_{2} \leq \cdots \leq d_{l} \leq d^{*}$ with the bounded value $d^{*}$, and $l$ is constant, representing the number of delayed states. $A, A_{d 1}, \ldots, A_{d l}$, and $B$ are known constant matrices with appropriate dimensions.
The structured uncertainties $[\Delta A, \Delta B]$ are time-varying matrices with appropriate dimensions, which are defined as follows:

$$
[\Delta A, \Delta B]=H \cdot F(\cdot)\left[E_{1}, E_{2}\right],
$$

where $H, E_{1}, E_{2}$ are known real constant matrices with appropriate dimensions. And $F(\cdot)$ is an unknown real- and time-varying matrix with Lebesgue-measurable elements satisfying

$$
F^{T}(\cdot) F(\cdot) \leq I
$$

It is assumed that the system (1) is stabilizable for the existence of a stabilizing feedback control and that the state $x(k)$ is available at every sampling instant $k$.

Lemma 1 (see [16]). Given any vectors $Z, Y \in \mathbb{R}^{n}$ and any positive definite symmetric matrices $P=P^{T} \in \mathbb{R}^{n \times n}$, such that

$$
2 Z^{T} P Y \leq Z^{T} P Z+Y^{T} P Y .
$$

Lemma 2 (see [16]). Given matrices $A, D, E, F$ with appropriate dimensions and for all $F$ satisfying $\|F\|<1$, then if there exist some positive definite symmetric matrices $P=P^{T}>0$ and $\varepsilon>0$ such that $P-\varepsilon D D^{T}>0$, then

$$
(A+D F E)^{T} P^{-1}(A+D F E)<A^{T}\left(P-\varepsilon D D^{T}\right)^{-1} A+\frac{1}{\varepsilon} E^{T} E .
$$

\section{Memory State Feedback Robust Model Predictive Controller}

In the section, we will focus on the controller synthesis for the linear system with multiple time-delayed states and the objective is to construct a memory state feedback controller as follows:

$$
\begin{aligned}
u(k+i \mid k)= & K(k) x(k+i \mid k) \\
& +\sum_{\tau=1}^{l} K_{d \tau}(k) x\left(k+i-d_{\tau} \mid k\right),
\end{aligned}
$$

where, $K(k), K_{d 1}(k), \ldots, K_{d l}(k)$ are gain matrices to be determined by MPC strategy. For the value of the state is measured at every sampling instant $k$, so we also assume that $d_{1}, d_{2}, \ldots, d_{l}$ are determined.

Firstly, we consider the following min-max optimization problem, which minimizes the worst case infinite horizon quadratic objective function:

$$
\min _{u(k+i \mid k)=K(k) x(k+i \mid k)+\sum_{\tau=1}^{l} K_{d \tau}(k) x\left(k+i-d_{\tau} \mid k\right)}\left[\max _{[A(k+i), B(k+i)], i>0} J(k)\right],
$$

where $Q_{1}, R$ are given positive definite symmetric matrices, and $J(k)=\sum_{i=0}^{\infty}\|x(k+i \mid k)\|_{Q_{1}}+\|u(k+i \mid k)\|_{R} \cdot x(k+i \mid k)$ and $u(k+i \mid k)$ are the predicted state and input, respectively, with $x(k \mid k)=x(k), x(k-i \mid k)=x(k-i)$ for $i \geq 0$. 
In order to design such a controller (6), we need to determine the upper bound of the cost (7). Now, based on Lyapunov-Krasovskii function, we define a delayed state dependent quadratic function as

$$
V(x(k))=\|x(k)\|_{P}+\sum_{\tau=1}^{l} \sum_{j=1}^{d_{\tau}}\|x(k-j)\|_{S_{\tau}},
$$

where $P=P^{T}>0, S_{\tau}=S_{\tau}^{T}>0(\tau=1, \ldots, l)$ are positive definite symmetric matrices and satisfy the following inequality for given structured uncertainties $[\Delta A, \Delta B]$ :

$$
\begin{aligned}
\Delta V(i, k) & =V(x(k+i+1 \mid k))-V(x(k+i \mid k)) \\
& \leq-\|x(k+i \mid k)\|_{\mathrm{Q}_{1}}-\|u(k+i \mid k)\|_{R} .
\end{aligned}
$$

Then, the cost monotonicity is guaranteed for the uncertain linear system (1). For the cost in (7) to be finite, we must have $V(x(\infty \mid k))=0$. Summing the both sides of inequality (9) from $i=1$ to $i=\infty$, we get an upper bound of the worst cost (7)

$$
\begin{aligned}
\max _{[\triangle A, \Delta B]=H F(\cdot)\left[E_{1}, E_{2}\right]} J(k) \leq & V(x(k \mid k)) \\
= & \|x(k \mid k)\|_{P} \\
& +\sum_{\tau=1}^{l} \sum_{j=1}^{d_{\tau}}\|x(k-j \mid k)\|_{S_{\tau}} .
\end{aligned}
$$

Therefore, the min-max problem is relaxed to the following minimization problem which minimizes the upper bound of the worst cost as

$$
\min _{P, S_{1}, \ldots, S_{l}}\left[\|x(k \mid k)\|_{P}+\sum_{\tau=1}^{l} \sum_{j=1}^{d_{\tau}}\|x(k-j \mid k)\|_{S_{\tau}}\right]
$$

subject to (1), (6), (9), and (10).

In order to convert the minimization problem (11) into an LMI optimization problem that can be efficiently solved by convex optimization algorithm, here we define the upper bound of the object function in (10) as

$$
\|x(k \mid k)\|_{P}+\sum_{\tau=1}^{l} \sum_{j=1}^{d_{\tau}}\|x(k-j \mid k)\|_{S_{\tau}} \leq \gamma(k),
$$

where $\gamma(k)$ is the nonnegative upper bound of $V$ to be minimized. Based on (12), the original min-max problem (11) can be redefined to be the following optimization problem that minimizes an upper bound on the worst value of the original cost function $J(k)$ :

$$
\min _{\gamma(k), P, S_{1}, \ldots, S_{l}} \gamma(k)
$$

subject to (1), (6), (9), and (12).

\section{Main Result}

First, we derive an LMI condition for the cost monotonicity (9) for the delayed state dependent quadratic function (8).

Theorem 3. The system (1) is asymptotically stable if there exist a symmetric positive definite matrix $Q=Q^{T}$, matrices $W, W_{1}, \ldots, W_{l}, Y, Y_{1}, \ldots, Y_{l}, \Lambda, \Lambda_{1}, \ldots, \Lambda_{l}$, and a constant $\gamma(k)>0$ satisfying the following LMI:

$$
\left[\begin{array}{ccccc}
u_{\max }^{2} I & * & * & * & * \\
Y^{T} & Q & * & * & * \\
Y_{1}^{T} & 0 & W_{1} & * & * \\
\vdots & \vdots & 0 & \ddots & * \\
Y_{l}^{T} & 0 & \cdots & 0 & W_{l}
\end{array}\right] \geq 0
$$

$$
\left[\begin{array}{ccccccc}
1 & * & * & * & * & * & * \\
x(k \mid k) & \mathrm{Q} & * & * & * & * & * \\
x(k-1 \mid k) & 0 & W_{1}+\cdots+W_{l} & * & * & * & * \\
\vdots & \vdots & \ddots & \ddots & * & * & * \\
x\left(k-d_{1}-1 \mid k\right) & 0 & 0 & 0 & W_{2}+\cdots+W_{l} & * & * \\
\vdots & \vdots & \vdots & \vdots & \ddots & \ddots & * \\
x\left(k-d_{l} \mid k\right) & 0 & 0 & 0 & 0 & 0 & W_{l}
\end{array}\right]>0
$$

$$
\left[\begin{array}{cccccccc}
\Phi_{1,1} & * & * & * & * & * & * & * \\
\Phi_{2,1} & \Phi_{2,2} & * & * & * & * & * & * \\
\Phi_{3,1} & 0 & \Phi_{3,3} & * & * & * & * & * \\
\Phi_{4,1} & 0 & 0 & \Phi_{4,4} & * & * & * & * \\
\vdots & \vdots & \vdots & \vdots & \ddots & * & * & * \\
\Phi_{l+3,1} & 0 & 0 & 0 & 0 & \Phi_{l+3, l+3} & * & * \\
\Phi_{l+4,1} & 0 & 0 & 0 & 0 & 0 & \Phi_{l+4, l+4} & * \\
\Phi_{l+5,1} & 0 & 0 & 0 & 0 & 0 & 0 & \Phi_{l+5, l+5}
\end{array}\right]>0
$$

$$
\left[\begin{array}{cc}
Q & * \\
H^{T} & \Lambda^{-1}
\end{array}\right]>0
$$

where $\Phi_{1,1}=(l+1)^{-1} Q, \Phi_{2,1}=Q_{1}^{0.5} Q, \Phi_{3,1}=R^{0.5} Y, \Phi_{4,1}=$ $\cdots=\Phi_{l+3,1}=Q, \Phi_{l+4,1}=E_{1} Q+E_{2} Y, \Phi_{l+5,1}=A Q+B Y$, $\Phi_{2,2}=(l+1) \gamma(k), \Phi_{3,3}=\gamma(k), \Phi_{4,4}=(l+1) W_{1}, \Phi_{l+3, l+3}=$ $(l+1) W_{l}, \Phi_{l+4, l+4}=\Lambda, \Phi_{l+5, l+5}=Q-\Lambda H H^{T}$.

Consider the following:

$$
\left[\begin{array}{cccc}
(l+1)^{-1} W_{\tau} & * & * & * \\
R^{0.5} Y_{\tau} & \gamma(k) & * & * \\
E_{2} Y_{\tau} & 0 & \Lambda_{\tau} & * \\
A_{d \tau} W_{\tau}+B Y_{\tau} & 0 & 0 & Q-\Lambda_{\tau} H H^{T}
\end{array}\right]>0
$$

$$
(\tau=1, \ldots, l),
$$

$$
\left[\begin{array}{cc}
Q & * \\
H^{T} & \Lambda_{\tau}^{-1}
\end{array}\right]>0 \quad(\tau=1, \ldots, l)
$$


Proof. Firstly, we derive the LMI condition for the inequality (12). The inequality (12) is rewritten as

$\|x(k \mid k)\|_{P}$

$$
+\left[\sum_{j=1}^{d_{1}}\|x(k-j \mid k)\|_{S_{1}}+\cdots+\sum_{j=1}^{d_{l}}\|x(k-j \mid k)\|_{S_{l}}\right]
$$

$\leq \gamma(k)$.

Multiplying both sides of the inequality (18a) by $\gamma^{-1}(k)$,

$$
\begin{aligned}
& \|x(k \mid k)\|_{\gamma^{-1}(k) P} \\
& +\left[\sum_{j=1}^{d_{1}}\|x(k-j \mid k)\|_{\gamma^{-1}(k) S_{1}}\right. \\
& \left.+\cdots+\sum_{j=1}^{d_{l}}\|x(k-j \mid k)\|_{\gamma^{-1}(k) S_{l}}\right]
\end{aligned}
$$

$\leq 1$.

Choosing $P=\gamma(k) Q^{-1}, S_{1}=\gamma(k) W_{1}^{-1}, \ldots, S_{l}=\gamma(k) W_{l}^{-1}$ and using Schur complement in [16], we have

$$
\left[\begin{array}{ccccccc}
1 & * & * & * & * & * & * \\
x(k \mid k) & \mathrm{Q} & * & * & * & * & * \\
x(k-1 \mid k) & 0 & W_{1}+\cdots+W_{l} & * & * & * & * \\
\vdots & \vdots & \ddots & \ddots & * & * & * \\
x\left(k-d_{1}-1 \mid k\right) & 0 & 0 & 0 & W_{2}+\cdots+W_{l} & * & * \\
\vdots & \vdots & \vdots & \vdots & \ddots & \ddots & * \\
x\left(k-d_{l} \mid k\right) & 0 & 0 & 0 & 0 & 0 & W_{l}
\end{array}\right]>0 .
$$

The differential of the quadratic function $V(x(k+j))$ is found by substituting (8) into (9),

$$
\begin{aligned}
\Delta V(i, k)= & V(x(k+i+1 \mid k))-V(x(k+i \mid k)) \\
= & \|x(k+i+1 \mid k)\|_{P}-\|x(k+i \mid k)\|_{P} \\
& +\|x(k+i \mid k)\|_{S_{1}}+\cdots+\|x(k+i \mid k)\|_{S_{l}} \\
& -\left\|x\left(k+i-d_{1} \mid k\right)\right\|_{S_{1}} \\
& -\cdots-\left\|x\left(k+i-d_{l} \mid k\right)\right\|_{S_{l}} \\
\leq & -\|x(k+i \mid k)\|_{\mathrm{Q}_{1}}-\|u(k+i \mid k)\|_{R} .
\end{aligned}
$$

From system (1) and the memory state feedback controller (6), we get

$$
\begin{aligned}
x & (k+i+1 \mid k) \\
= & A(k+i) x(k+i \mid k) \\
& +\sum_{\tau=1}^{l} A_{d \tau} x\left(k+i-d_{\tau} \mid k\right)+B(k+i) \\
& \times\left[K(k) x(k+i \mid k)+\sum_{\tau=1}^{l} K_{d \tau}(k) x\left(k+i-d_{\tau} \mid k\right)\right] \\
= & {[A(k+i)+B(k+i) K(k)] x(k+i \mid k) } \\
& +\sum_{\tau=1}^{l}\left[A_{d \tau}+B(k+i) K_{d \tau}(k)\right] x\left(k+i-d_{\tau} \mid k\right) \\
= & A_{K} x(k+i \mid k)+\sum_{\tau=1}^{l} A_{K d \tau} x\left(k+i-d_{\tau} \mid k\right),
\end{aligned}
$$

where $A_{K d \tau}=A_{d \tau}+B(k+i) K_{d \tau}(k), A_{K}=A(k+i)+B(k+$ i) $K(k)$. Then, we obtain $\|x(k+i+1 \mid k)\|_{P}$ as follows:

$$
\|x(k+i+1 \mid k)\|_{P}
$$

$$
=\left\|A_{K} x(k+i \mid k)+\sum_{\tau=1}^{l} A_{K d \tau} x\left(k+i-d_{\tau} \mid k\right)\right\|_{P} .
$$

Substituting (22) into (20), we have

$$
\begin{aligned}
\Delta V(i, k)= & \left\|A_{K} x(k+i \mid k)+\sum_{\tau=1}^{l} A_{K d \tau} x\left(k+i-d_{\tau} \mid k\right)\right\|_{P} \\
& -\|x(k+i \mid k)\|_{P}+\|x(k+i \mid k)\|_{S_{1}} \\
& +\cdots+\|x(k+i \mid k)\|_{S_{l}}-\left\|x\left(k-d_{1}+i \mid k\right)\right\|_{S_{1}} \\
& -\cdots-\left\|x\left(k-d_{l}+i \mid k\right)\right\|_{S_{l}} \\
\leq & -\|x(k+i \mid k)\|_{Q_{1}}-\|u(k+i \mid k)\|_{R} .
\end{aligned}
$$

Then, the inequality (23) in matrix form is

$$
\begin{aligned}
\Delta V(i, k) & =\mathbf{X}^{T}\left[\begin{array}{cccc}
\Xi_{1,1} & * & * & * \\
\Xi_{2,1} & \Xi_{2,2} & * & * \\
\vdots & \vdots & \ddots & * \\
\Xi_{l+1,1} & \Xi_{1+1,2} & \cdots & \Xi_{l+1, l+1}
\end{array}\right] \mathbf{X} \\
& \leq-\|x(k+i \mid k)\|_{Q_{1}}-\|u(k+i \mid k)\|_{R},
\end{aligned}
$$

where $\Xi_{1,1}=A_{K}^{T} P A_{K}-P+S_{1}+\cdots+S_{l}, \Xi_{1,2}=$ $A_{K}^{T} P A_{K d 1}, \Xi_{1, l+1}=A_{K}^{T} P A_{K d l}, \Xi_{2,1}=A_{K d 1}^{T} P A_{K}, \Xi_{2, l+1}=$ $A_{K d 1}^{T} P A_{K d l}, \Xi_{l+1,1}=A_{K d l}^{T} P A_{K}, \Xi_{l+1,2}=A_{K d l}^{T} P A_{K d 1}$, $\Xi_{2,2}=A_{K d l}^{T} P A_{K d 1}-S_{1}, \Xi_{l+1, l+1}=A_{K d l}^{T} P A_{K d l}-S_{l}, \mathbf{X}^{T}=$ $\left[\begin{array}{lllll}x(k+i \mid k) & x\left(k+i-d_{1} \mid k\right) & \cdots & x\left(k+i-d_{l} \mid k\right)\end{array}\right]^{T}$. 
From (6), we can obtain

$$
\begin{aligned}
& \|u(k+i \mid k)\|_{R} \\
& =\left\|K(k) x(k+i \mid k)+\sum_{\tau=1}^{l} K_{d \tau}(k) x\left(k+i-d_{\tau} \mid k\right)\right\|_{R} \\
& =\mathbf{X}^{T}\left[\begin{array}{ccccc}
\Psi_{1,1} & \Psi_{1,2} & \Psi_{1,3} & \cdots & \Psi_{1, l+1} \\
\Psi_{2,1} & \Psi_{2,2} & \Psi_{2,3} & \cdots & \Psi_{2, l+1} \\
\Psi_{3,1} & \Psi_{3,2} & \Psi_{3,3} & \cdots & \Psi_{3, l+1} \\
\vdots & \vdots & \vdots & \ddots & \vdots \\
\Psi_{l+1,1} & \Psi_{l+1,2} & \Psi_{l+1,3}^{\prime} & \cdots & \Psi_{l+1, l+1}^{\prime}
\end{array}\right] \mathbf{X} \\
& =\mathbf{X}^{T}\left[\begin{array}{c}
K^{T} \\
K_{d 1}^{T} \\
\vdots \\
K_{d 1}^{T}
\end{array}\right] R\left[\begin{array}{llll}
K & K_{d 1} & \cdots & K_{d l}
\end{array}\right] \mathbf{X},
\end{aligned}
$$

where $\Psi_{1,1}=K^{T} R K, \Psi_{2,1}=K^{T} R K_{d 1}, \Psi_{3,1}=$ $K^{T} R K_{d 2}, \Psi_{l+1,1}=K^{T} R K_{d l}, \Psi_{1,2}=K_{d 1}^{T} R K, \Psi_{2,2}=K_{d 1}^{T} R K_{d 1}$, $\Psi_{3,2}=K_{d 1}^{T} R K_{d 2}, \Psi_{l+1,2}=K_{d 1}^{T} R K_{d l}, \Psi_{3,1}=K_{d 2}^{T} R K, \Psi_{3,2}=$ $K_{d 2}^{T} R K_{d 1}, \Psi_{3,3}=K_{d 2}^{T} R K_{d 2}, \Psi_{l+1,3}=K_{d 2}^{T} R K_{d l}, \Psi_{l+1,1}=K_{d l}^{T} R K$, $\Psi_{l+1,2}=K_{d l}^{T} R K_{d 2}, \Psi_{l+1,3}=K_{d l}^{T} R K_{d 3}, \Psi_{l+1, l+1}=K_{d l}^{T} R K_{d l}$.

Substituting (22) into (20) and from (24), the following holds:

$$
\begin{aligned}
& {\left[\begin{array}{cccc}
\Xi_{1,1} & \Xi_{1,2} & \cdots & \Xi_{1, l+1} \\
\Xi_{2,1} & \Xi_{2,2} & \cdots & \Xi_{2, l+1} \\
\vdots & \vdots & \ddots & \vdots \\
\Xi_{l+1,1} & \Xi_{1+1,2} & \cdots & \Xi_{l+1, l+1}
\end{array}\right]} \\
& =\left[\begin{array}{ccccc}
M_{00} & * & * & * & * \\
M_{10} & M_{11} & * & * & * \\
M_{20} & M_{21} & M_{22} & * & * \\
\vdots & \vdots & \vdots & \ddots & * \\
M_{l 0} & M_{l 1} & M_{l 2} & \cdots & M_{l l}
\end{array}\right] \\
& +\left[\begin{array}{cccc}
Q_{1}-P+S_{1}+\cdots+S_{l} & * & * & * \\
0 & -S_{1} & * & * \\
\vdots & \vdots & \ddots & * \\
0 & 0 & \cdots & -S_{l}
\end{array}\right]=\bar{M}^{\prime}+M^{\prime} \text {, }
\end{aligned}
$$

where $M_{j \varsigma}=K_{d j}^{T} R K_{d \varsigma}+A_{K d j}^{T} P A_{K d \varsigma}(j=0,1, \ldots, l, \varsigma=$ $0,1, \ldots, l), K_{d 0}=K, A_{K d 0}=A_{K}$.

From (24) and (26), we have

$$
\begin{aligned}
\mathbf{X}^{T} \bar{M}^{\prime} \mathbf{X}= & \sum_{\varsigma=0}^{l} \sum_{j=0}^{l} x^{T}\left(k+i-d_{j} \mid k\right) \\
& \times\left[K_{d j}^{T} R K_{d \varsigma}+A_{K d j}{ }^{T} P A_{K d \varsigma}\right] \\
& \times x\left(k+i-d_{\varsigma} \mid k\right) .
\end{aligned}
$$

By Lemma 1, the following holds:

$$
\begin{aligned}
x^{T}( & \left.+i-d_{j} \mid k\right)\left[K_{d j}^{T} R K_{d \varsigma}+A_{K d j}^{T} P A_{K d \varsigma}\right] \\
& \times x\left(k+i-d_{\varsigma} \mid k\right) \\
= & {\left[K_{d j} x\left(k+i-d_{j} \mid k\right)\right]^{T} R\left[K_{d \varsigma} x\left(k+i-d_{\varsigma} \mid k\right)\right] } \\
& +\left[A_{K d j} x\left(k+i-d_{j} \mid k\right)\right]^{T} P\left[A_{K d \varsigma} x\left(k+i-d_{\varsigma} \mid k\right)\right] \\
\leq & \frac{1}{2}\left(\left\|K_{d j} x\left(k+i-d_{j} \mid k\right)\right\|_{R}+\left\|K_{d \varsigma} x\left(k+i-d_{\varsigma} \mid k\right)\right\|_{R}\right. \\
& \left.+\left\|A_{K d j} x\left(k+i-d_{j} \mid k\right)\right\|_{P}+\left\|A_{K d \varsigma} x\left(k+i-d_{\varsigma} \mid k\right)\right\|_{P}\right) .
\end{aligned}
$$

Then, inequality (27) can be derived as in the following form:

$\mathbf{X}^{T} \bar{M}^{\prime} \mathbf{X}$

$$
\begin{aligned}
& \leq \frac{1}{2} \sum_{\varsigma=0}^{l} \sum_{j=0}^{l}\left\|K_{d j} x\left(k+i-d_{j} \mid k\right)\right\|_{R} \\
& +\left\|K_{d \varsigma} x\left(k+i-d_{\varsigma} \mid k\right)\right\|_{R} \\
& +\left\|A_{K d j} x\left(k+i-d_{j} \mid k\right)\right\|_{P}+\left\|A_{K d \varsigma} x\left(k+i-d_{\varsigma} \mid k\right)\right\|_{P} \\
& =(l+1) X^{T}\left[\begin{array}{ccccc}
\Pi_{0,0} & * & * & * & * \\
0 & \Pi_{1,1} & * & * & * \\
0 & 0 & \Pi_{2,2} & * & * \\
\vdots & \vdots & \vdots & \ddots & * \\
0 & 0 & 0 & 0 & \Pi_{l, l}
\end{array}\right] X \text {, }
\end{aligned}
$$

where $\Pi_{0,0}=K^{T} R K+A_{K}^{T} P A_{K}, \Pi_{1,1}=K_{d 1}^{T} R K_{d 1}+A_{d q}^{T} P A_{K d 1}$, $\Pi_{2,2}=K_{d 2}^{T} R K_{d 2}+A_{K d 2}^{T} P A_{K d 2}, \Pi_{l, l}=K_{d l}^{T} R K_{d l}+A_{K d l}^{T} P A_{K d l}$. Therefore,

$\Delta V(x(k+i \mid k))$

$$
\begin{gathered}
\leq X^{T}\left[(l+1)\left[\begin{array}{ccccc}
\Pi_{0,0} & * & * & * & * \\
0 & \Pi_{1,1} & * & * & * \\
0 & 0 & \Pi_{2,2} & * & * \\
\vdots & \vdots & \vdots & \ddots & * \\
0 & 0 & 0 & 0 & \Pi_{l, l}
\end{array}\right]\right. \\
\left.+\left[\begin{array}{cccccc}
Q_{1}-P+S_{1}+\cdots+S_{l} & * & * & * & * \\
0 & & -S_{1} & * & * & * \\
0 & & 0 & -S_{2} & * & * \\
\vdots & & \vdots & 0 & \ddots & * \\
0 & & 0 & \cdots & 0 & -S_{3}
\end{array}\right]\right] X .
\end{gathered}
$$


Thus, inequality (30) is satisfied if

$$
\begin{gathered}
(l+1)\left(K^{T} R K+A_{K}^{T} P A_{K}\right)+Q_{1}-P+S_{1}+\cdots+S_{l} \leq 0 \\
(l+1)\left(K_{d 1}^{T} R K_{d 1}+A_{K d 1}^{T} P A_{K d 1}\right)-S_{1} \leq 0 \\
\vdots \\
(l+1)\left(K_{d l}^{T} R K_{d l}+A_{K d l}^{T} P A_{K d l}\right)-S_{l} \leq 0
\end{gathered}
$$

holds.

Substituting $A_{K d \tau}=A_{d \tau}+(B+\Delta B) K_{d \tau}, A_{K}=A+\Delta A+$ $(B+\Delta B) K(\tau=1, \ldots, l)$, and (2) into (31), by Lemma 2, we can obtain

$$
\begin{aligned}
(l+1) & \left(K^{T} R K+A_{K}^{T} P A_{K}\right)+Q_{1}-P+S_{1}+\cdots+S_{l} \\
= & (l+1)\left[A+B K+H F(k)\left(E_{1}+E_{2} K\right)\right]^{T} \\
& \times P\left[A+B K+H F(k)\left(E_{1}+E_{2} K\right)\right] \\
& +(l+1) K^{T} R K+Q_{1}-P+S_{1}+\cdots+S_{l} \\
\leq & (l+1)\left[(A+B K)^{T}\left(P-\varepsilon H H^{T}\right)^{-1}(A+B K)\right. \\
& +(l+1) K^{T} R K+Q_{1}-P+S_{1}+\cdots+S_{l} \leq 0 \\
& \left.(l+1)\left[A_{d \tau}+B K_{d \tau}+H F(k)\left(E_{2} K_{d \tau}\right)\right]^{T}\left(E_{1}+E_{2} K\right)\right] \\
& \times P\left[A_{d \tau}+B K_{d \tau}+H F(k)\left(E_{2} K_{d \tau}\right)\right] \\
& +(l+1) K_{d \tau}^{T} R K_{d \tau}-S_{\tau} \leq 0 \quad(\tau=1, \ldots, l)
\end{aligned}
$$

Multiplying both sides of the inequality (32a) by $Q^{T}$ and $(l+1)^{-1} \gamma^{-1} \mathrm{Q}$, it equals the following inequality:

$$
\begin{aligned}
& {\left[(A Q+B K Q)^{T}\left(\gamma P^{-1}-\gamma \varepsilon H^{T} H\right)^{-1}(A Q+B K Q)\right.} \\
& \left.+\frac{1}{\gamma \varepsilon}\left(E_{1} Q+E_{2} K Q\right)^{T}\left(E_{1} Q+E_{2} K Q\right)\right] \\
& +\frac{1}{\gamma} \times(K Q)^{T} R(K Q)+Q^{T} \frac{Q_{1}}{(l+1) \gamma} Q-Q^{T} \frac{P}{(l+1) \gamma} Q \\
& +Q^{T} \frac{S_{1}}{(l+1) \gamma} Q+\cdots+Q^{T} \frac{S_{l}}{(l+1) \gamma} Q \leq 0 .
\end{aligned}
$$

Now, choose $P^{-1}=\gamma^{-1} Q, S_{1}=\gamma^{-1} W_{1}, \ldots, S_{l}=\gamma^{-1} W_{l}$, $\Lambda=\gamma \varepsilon, K Q=Y$; then by using Schur complement [16], the inequality (32a) can be derived as the following inequalities:

$$
\begin{gathered}
{\left[\begin{array}{cccccccc}
\Phi_{1,1} & * & * & * & * & * & * & * \\
\Phi_{2,1} & \Phi_{2,2} & * & * & * & * & * & * \\
\Phi_{3,1} & 0 & \Phi_{3,3} & * & * & * & * & * \\
\Phi_{4,1} & 0 & 0 & \Phi_{4,4} & * & * & * & * \\
\vdots & \vdots & \vdots & \vdots & \ddots & * & * & * \\
\Phi_{l+3,1} & 0 & 0 & 0 & 0 & \Phi_{l+3, l+3} & * & * \\
\Phi_{l+4,1} & 0 & 0 & 0 & 0 & 0 & \Phi_{l+4, l+4} & * \\
\Phi_{l+5,1} & 0 & 0 & 0 & 0 & 0 & 0 & \Phi_{l+5, l+5}
\end{array}\right]>0,} \\
\\
{\left[\begin{array}{ccc}
Q & * \\
H^{T} & \Lambda^{-1}
\end{array}\right]>0,}
\end{gathered}
$$

where $\Phi_{1,1}=(l+1)^{-1} Q, \Phi_{2,1}=Q_{1}^{0.5} Q, \Phi_{3,1}=R^{0.5} Y, \Phi_{4,1}=$ $\cdots=\Phi_{l+3,1}=Q, \Phi_{l+4,1}=E_{1} Q+E_{2} Y, \Phi_{l+5,1}=A Q+B Y$, $\Phi_{2,2}=(l+1) \gamma, \Phi_{3,3}=\gamma, \Phi_{4,4}=(l+1) W_{1}, \Phi_{l+3, l+3}=(l+1) W_{l+3}$, $\Phi_{l+4, l+4}=\Lambda, \Phi_{l+5, l+5}=\mathrm{Q}-\Lambda H H^{T}$.

Multiplying both sides of the inequality (32b) by $W_{\tau}^{T}$ and $(l+1)^{-1} \gamma^{-1} W_{\tau}$ and by Lemma 2 , it equals the following inequality:

$$
\begin{aligned}
\left(A_{d \tau} W_{\tau}+B K_{d \tau} W_{\tau}\right)^{T}\left(\gamma P^{-1}-\gamma \varepsilon_{\tau} H H^{T}\right)^{-1} \\
\quad \times\left(A_{d \tau} W_{\tau}+B K_{d \tau} W_{\tau}\right) \\
\quad+\left(\gamma \varepsilon_{\tau}\right)^{-1}\left(E_{3} K_{d \tau} W_{\tau}\right)^{T}\left(E_{3} K_{d \tau} W_{\tau}\right) \\
\quad+\left(K_{d \tau} W_{\tau}\right)^{T} R \gamma^{-1}\left(K_{d \tau} W_{\tau}\right)-(l+1)^{-1} W_{\tau} \leq 0 .
\end{aligned}
$$

Now, choose $S_{1}=\gamma^{-1} W_{1}, \ldots, S_{l}=\gamma^{-1} W_{l}, \Lambda_{\tau}=\gamma \varepsilon_{\tau}, K_{d \tau} W_{\tau}=$ $Y_{\tau}$, then by using Schur complement [16], the inequality (35) can be derived as following inequalities:

$$
\begin{gathered}
{\left[\begin{array}{cccc}
(l+1)^{-1} W_{\tau} & * & * & * \\
R^{0.5} Y_{\tau} & \gamma & * & * \\
E_{3} Y_{\tau} & 0 & \Lambda_{\tau} & * \\
A_{d \tau} W_{\tau}+B Y_{\tau} & 0 & 0 & Q-\Lambda_{\tau} H H^{T}
\end{array}\right]>0} \\
\\
{\left[\begin{array}{cc}
Q & * \\
H^{T} & \Lambda_{\tau}^{-1}
\end{array}\right]>0 .}
\end{gathered}
$$

Secondly, we convert the input constraint (1) into LMI form. From (12), we obtain

$$
\|x(k+i \mid k)\|_{P}+\sum_{\tau=1}^{l} \sum_{j=1}^{d_{\tau}}\|x(k+i-j \mid k)\|_{S_{\tau}} \leq \gamma(k), \quad i \geq 1 .
$$

Since $\gamma>0$, then inequality (37) can be rewritten as

$$
\begin{aligned}
x^{T}(k & +i \mid k) \gamma^{-1} P x(k+i \mid k) \\
& +\sum_{\tau=1}^{l} x^{T}\left(k+i-d_{\tau} \mid k\right) \gamma^{-1} S_{\tau} x\left(k+i-d_{\tau} \mid k\right)<1 .
\end{aligned}
$$


For $Q=\gamma P^{-1}>0, W_{1}=\gamma S_{1}^{-1}>0, \ldots, W_{l}=\gamma S_{l}^{-1}>0$, then we have

$$
\begin{aligned}
x^{T}(k+i \mid k) Q x(k+i \mid k) & \\
& +\sum_{\tau=1}^{l} x^{T}\left(k+i-d_{\tau} \mid k\right) W_{\tau} x\left(k+i-d_{\tau} \mid k\right)<1 .
\end{aligned}
$$

Moreover, $Q>0, W_{\tau}>0$, we can obtain that

$$
\begin{gathered}
x^{T}(k+i \mid k) Q x(k+i \mid k)<1 \\
x^{T}\left(k+i-d_{1} \mid k\right) W_{1} x\left(k+i-d_{1} \mid k\right)<1 \\
\vdots \\
x^{T}\left(k+i-d_{\tau} \mid k\right) W_{\tau} x\left(k+i-d_{\tau} \mid k\right)<1 .
\end{gathered}
$$

From (6), $K=Y Q^{-1}$ and $K_{d \tau}=Y_{\tau} W_{\tau}^{-1}(\tau=1, \ldots, l)$, the input constraint can be expressed as

$$
\begin{aligned}
\|u(k+i \mid k)\|_{2} \leq & u_{\max } \\
\Longleftrightarrow & \Longleftrightarrow K x(k+i \mid k)+\sum_{\tau=1}^{l} K_{d \tau} x\left(k+i-d_{\tau} \mid k\right) \|_{2} \\
\leq & u_{\max } \\
\Longleftrightarrow & \|K x(k+i \mid k)\|_{2}+\left\|K_{d 1} x\left(k+i-d_{1} \mid k\right)\right\|_{2} \\
& +\cdots+\left\|K_{d l} x\left(k+i-d_{l} \mid k\right)\right\|_{2} \leq u_{\max } \\
\Longleftrightarrow & \left\|Y Q^{-1} x(k+i \mid k)\right\|_{2} \\
& +\left\|Y_{1} W_{1}^{-1} x\left(k+i-d_{1} \mid k\right)\right\|_{2} \\
& +\cdots+\left\|Y_{l} W_{l}^{-1} x\left(k+i-d_{l} \mid k\right)\right\|_{2} \leq u_{\max } .
\end{aligned}
$$

The inequality (39) is satisfied if

$$
\left[\begin{array}{ccccc}
u_{\max }^{2} I & * & * & * & * \\
Y^{T} & Q & * & * & * \\
Y_{1}^{T} & 0 & W_{1} & * & * \\
\vdots & \vdots & 0 & \ddots & * \\
Y_{l}^{T} & 0 & \cdots & 0 & W_{l}
\end{array}\right] \geq 0
$$

holds. This completes the proof.

Now, the minimization problem (13) is converted to the following LMI optimization problem:

$$
\min _{\gamma(k), P, W_{1}, \ldots, W_{l}, Y, Y_{1}, \ldots, Y_{l}} \gamma(k)
$$

subject to (14), (15), (16a), and (16b).

Theorem 4 (closed loop stability). If the optimization problem (43) is feasible at the initial instant $k=0$, then the proposed robust memory state feedback MPC law (6) stabilizes the system (1) asymptotically.
Proof. Let $P(k)$ and $P(k+1)$ denote the optimal solutions of the optimization problem (43) at instant $k$ and $k+1$, respectively. Let us consider a quadratic function

$$
V(x(k))=\|x(k)\|_{P(k)}+\sum_{\tau=1}^{l} \sum_{j=1}^{d_{\tau}}\|x(k-j)\|_{S_{\tau}} .
$$

Since $P(k+1)$ is optimal, while $P(k)$ is only feasible at instant $k+1$,

$$
\begin{aligned}
& V(x(k+1)) \\
& =\|x(k+1)\|_{P(k+1)}+\sum_{\tau=1}^{l} \sum_{j=1}^{d_{\tau}}\|x(k-j+1)\|_{S_{\tau}} \\
& \leq\|x(k+1)\|_{P(k)}+\sum_{\tau=1}^{l} \sum_{j=1}^{d_{\tau}}\|x(k-j+1)\|_{S_{\tau}} .
\end{aligned}
$$

Besides, it follows from (9) that

$$
\begin{gathered}
\|x(k+1 \mid k)\|_{P(k)}+\sum_{\tau=1}^{l} \sum_{j=1}^{d_{\tau}}\|x(k-j+1 \mid k)\|_{S_{\tau}} \\
\leq\|x(k \mid k)\|_{P(k)}+\sum_{\tau=1}^{l} \sum_{j=1}^{d_{\tau}}\|x(k-j \mid k)\|_{S_{\tau}} \\
-\left[\|x(k \mid k)\|_{Q_{1}}+\|u(k \mid k)\|_{R}\right]
\end{gathered}
$$

for any structured uncertainties $[\Delta A, \Delta B]$.

Since $x(k+1 \mid k+1)$ equals $A(k) x(k \mid k)+\sum_{\tau=1}^{l} A_{d \tau} x(k-$ $\left.d_{\tau} \mid k\right)+B(k) u(k \mid k)$ for given uncertainties, (46) must hold with $x(k+1 \mid k+1)$ in place of $x(k+1 \mid k)$. Combining this with (45), we have

$$
\begin{aligned}
& V(x(k+1 \mid k+1)) \\
& \quad \leq V(x(k \mid k))-\left[\|x(k \mid k)\|_{\mathrm{Q}_{1}}+\|u(k \mid k)\|_{R}\right] .
\end{aligned}
$$

Summing inequality (47) from $k=0$ to $k=i-1$ yields

$$
\begin{aligned}
& V(x(i \mid i))+\sum_{k=0}^{i-1}\left[\|x(k \mid k)\|_{\mathrm{Q}_{1}}+\|u(k \mid k)\|_{R}\right] \\
& \leq V(x(0 \mid 0)) .
\end{aligned}
$$

Since $V(x(i \mid i)) \geq 0$ and the left hand side of (48) is bounded above by the constant $V(x(0 \mid 0)), x(i \mid i)$ and $u(i \mid i)$ must go to zero as $i$ goes to infinity. Therefore, the closed loop stability is guaranteed.

\section{Numerical Example}

In this section, a numerical example is presented to illustrate the effectiveness of the proposed memory state feedback robust MPC algorithm. Let us consider the following discrete time multiple time-delayed uncertain linear system:

$$
x(k+1)=A(k) x(k)+\sum_{\tau=1}^{l} A_{d \tau} x\left(k-d_{\tau}\right)+B(k) u(k),
$$



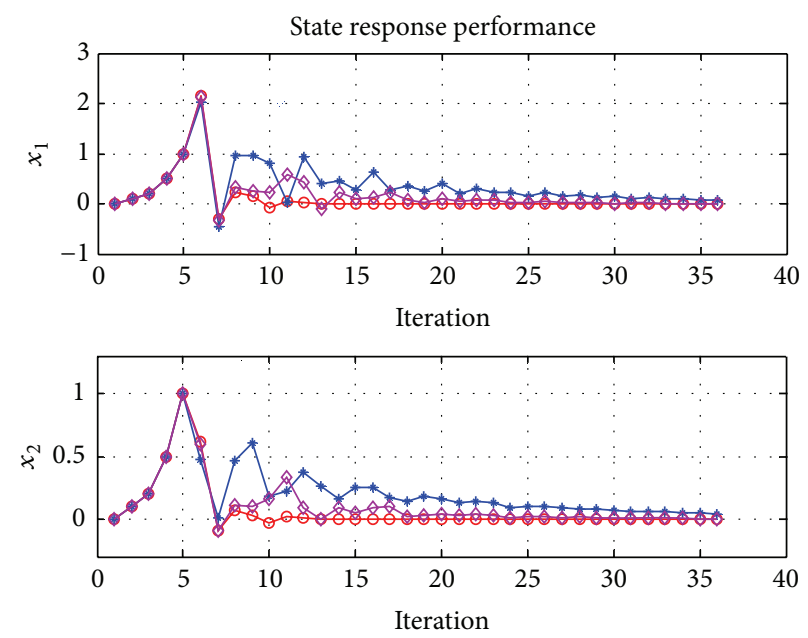

- - The multiple time-delayed state memory controller in this paper

$\rightarrow$ The memoryless controller obtained by Kothare's method

$\rightarrow$ The controller designed using the algorithm of [13]

FIGURE 1: State responses for $x_{1}$ and $x_{2}$.

where $A=\left[\begin{array}{ll}0.0 & 1.2 \\ 1.0 & -1.5\end{array}\right], A_{d 1}=\left[\begin{array}{ll}0.2 & 0.2 \\ 0.2 & 0.1\end{array}\right], A_{d 2}=\left[\begin{array}{ll}0.02 & 0.02 \\ 0.02 & 0.01\end{array}\right]$, $B=\left[\begin{array}{ll}1.1 & 1.3\end{array}\right]^{T}, E_{1}=\left[\begin{array}{ll}0.2 & 0.3\end{array}\right], E_{2}=0.9, F=\sin k$, $H=\left[\begin{array}{ll}0.1 & 0.1\end{array}\right]^{T}$.

The initial value state is given as $x(0)=[1,1]^{T}$; the state and the input weighting matrices are $Q_{1}=\operatorname{diag}(1,1)$ and $R=$ 0.05 . The delay indexes of the system are $d_{1}=2, d_{2}=4, l=2$ and control constraint is $|u| \leq 2$.

The following terminology is used for the controllers designed:

(i) $u(k+i \mid k)=K(k) x(k+i \mid k)$, the controller is referred to as a memoryless controller obtained by Kothare's method;

(ii) $u(k+i \mid k)=K(k) x(k+i \mid k)+K_{d 1}(k) x\left(k+i-d_{1} \mid k\right)$, the controller is referred to as a memory controller with a single time-delayed state $x\left(k-d_{1} \mid k\right)$ obtained by Qin's method and ZHAO's method;

(iii) $u(k+i \mid k)=K(k) x(k+i \mid k)+\sum_{\tau=1}^{l} K_{d \tau}(k) x(k+$ $\left.i-d_{\tau} \mid k\right)$, the controller is referred to as a multiple time-delayed state memory controller in this paper.

The simulation results are compared with the results that were with a memoryless state feedback controller [4] and Qin' method with a memory state feedback controller with single time-delay state. (The time-delayed state $x\left(k-d_{1}\right)$ was used to design control input, which was more severe than the time- delayed state $\left.x\left(k-d_{2}\right)\right)$. In Figure 1, the stabilization performances for the three techniques are also compared. From Figure 2, the inputs are within their limits and no saturation is observed all the time. Moreover, Figure 3 shows that the cost function is smaller than the one obtained by the technique of a memoryless state feedback controller and the controller using the method in [13]. Hence, we can see that the improvement is more evident in Figures 1 and 3. In the

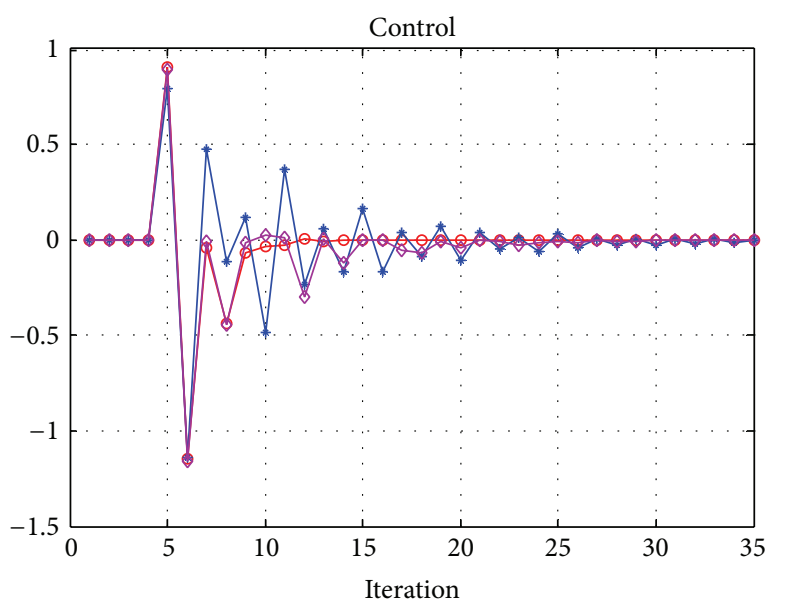

$\rightarrow$ The multiple time-delayed state memory controller in this paper
$\rightarrow$ The memoryless controller obtained by Kothare's method
$\rightarrow$ The controller designed using the algorithm of [13]

FIGURE 2: Control input.

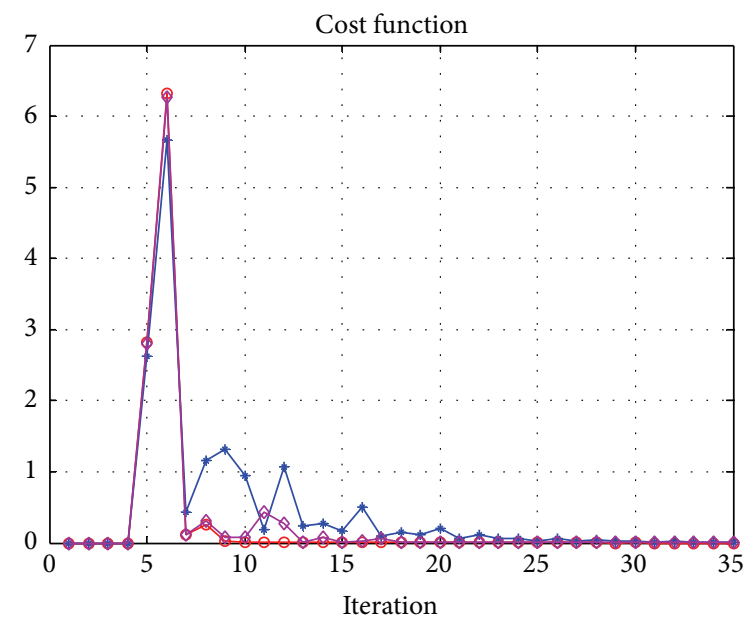

$\rightarrow$ The multiple time-delayed state memory controller in this paper
$\rightarrow$ The memoryless controller obtained by Kothare's method
$\rightarrow$ The controller designed using the algorithm of [13]

Figure 3: Cost index.

simulation results, stabilization performance as well as the cost is improved by using the memory state feedback RMPC.

\section{Conclusions}

In this paper, a new robust memory state feedback MPC technique was proposed for a class of multiple time-delayed uncertain linear systems with input constraints. The minimization problem for infinite horizon cost was derived using the time-delayed state dependent Lyapunov function. The optimization problem was reformulated in the form of a finite number of LMIs. Numerical examples demonstrated the effectiveness of the proposed method. 


\section{Conflict of Interests}

The authors declare that there is no conflict of interests regarding the publication of this paper.

\section{Acknowledgments}

This work is supported in part by the National Natural Science Foundation (NNSF) of China under Grants 61304001 and 61203007. The authors would also like to thank He Bin and $\mathrm{Hu}$ Xiao-xiang for Matlab simulations.

\section{References}

[1] D. Q. Mayne, J. B. Rawlings, C. V. Rao, and P. O. M. Scokaert, "Constrained model predictive control: stability and optimality," Automatica. A Journal of IFAC, the International Federation of Automatic Control, vol. 36, no. 6, pp. 789-814, 2000.

[2] Y. G. Xi and D. W. Li, "Fundamental philosophy and status of qualitative synthesis of model predictive control," Acta Automatica Sinica, vol. 34, no. 10, pp. 1225-1234, 2008.

[3] Y.-G. Xi, D.-W. Li, and S. Lin, "Model predictive control-current status and challenges," Acta Automatica Sinica, vol. 39, no. 3, pp. 222-236, 2013.

[4] M. V. Kothare, V. Balakrishnan, and M. Morari, "Robust constrained model predictive control using linear matrix inequalities," Automatica. A Journal of IFAC, the International Federation of Automatic Control, vol. 32, no. 10, pp. 1361-1379, 1996.

[5] W. H. Kwon, J. W. Kang, Y. S. Lee, and Y. S. Moon, "A simple receding horizon control for state delayed systems and its stability criterion," Journal of Process Control, vol. 13, no. 6, pp. 539-551, 2003.

[6] P.-L. Liu, "State feedback stabilization of time-varying delay uncertain systems: a delay decomposition approach," Linear Algebra and its Applications, vol. 438, no. 5, pp. 2188-2209, 2013.

[7] S. C. Jeong and P. Park, "Constrained MPC algorithm for uncertain time-varying systems with state-delay," Institute of Electrical and Electronics Engineers. Transactions on Automatic Control, vol. 50, no. 2, pp. 257-263, 2005.

[8] C. Briat, O. Sename, and J. F. Lafay, "Memory-resilient gainscheduled state-feedback control of uncertain LTI/LPV systems with time-varying delays," Systems \& Control Letters, vol. 59, no. 8, pp. 451-459, 2010.

[9] F. Gouaisbaut and D. Peaucelle, "Delay-dependent robust stability of time delay systems," in Proceedings of the 5th IFAC Symposium on Robust Control Design (ROCOND '06), pp. 453458, Toulouse, France, July 2006.

[10] C.-Y. Kao and A. Rantzer, "Stability analysis of systems with uncertain time-varying delays," Automatica. A Journal of IFAC, the International Federation of Automatic Control, vol. 43, no. 6 , pp. 959-970, 2007.

[11] D. H. Ji, J. H. Park, W. J. Yoo, and S. C. Won, "Robust memory state feedback model predictive control for discretetime uncertain state delayed systems," Applied Mathematics and Computation, vol. 215, no. 6, pp. 2035-2044, 2009.

[12] R. Dey, S. Ghosh, G. Ray, and A. Rakshit, "State feedback stabilization of uncertain linear time-delay systems: a nonlinear matrix inequality approach," Numerical Linear Algebra with Applications, vol. 18, no. 3, pp. 351-361, 2011.
[13] Q. Wei-Wei, L. Gang, and Z. Zhiqiang, "Memory state feedbackbased model predictive controller of time-delay systems with input constraints," Journal of South China University of Technology (Natural Science Edition), vol. 40, no. 6, pp. 63-68, 2012.

[14] C.-L. Su, J.-C. Zhao, and P. Li, "Robust predictive control for a class of multiple time delay uncertain systems with nonlinear disturbance," Acta Automatica Sinica, vol. 39, no. 5, pp. 644-649, 2013.

[15] J.-C. Zhao, Y.-M. Fang, and L. I. Jian-Xiong, "Robust predictive control for a class of uncertain discrete system with multiple state delays," in Proceedings of the 30th Chinese Control Conference (CCC '11), pp. 3372-3376, July 2011.

[16] Z. D. Wang, B. Huang, and H. Unbehauen, "Robust reliable control for a class of uncertain nonlinear state-delayed systems," Automatica. A Journal of IFAC, the International Federation of Automatic Control, vol. 35, no. 5, pp. 955-963, 1999. 


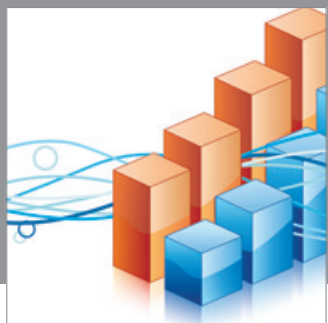

Advances in

Operations Research

mansans

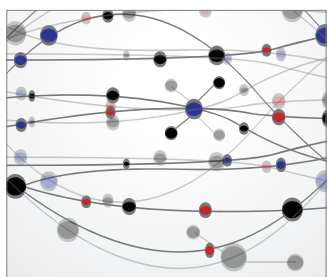

The Scientific World Journal
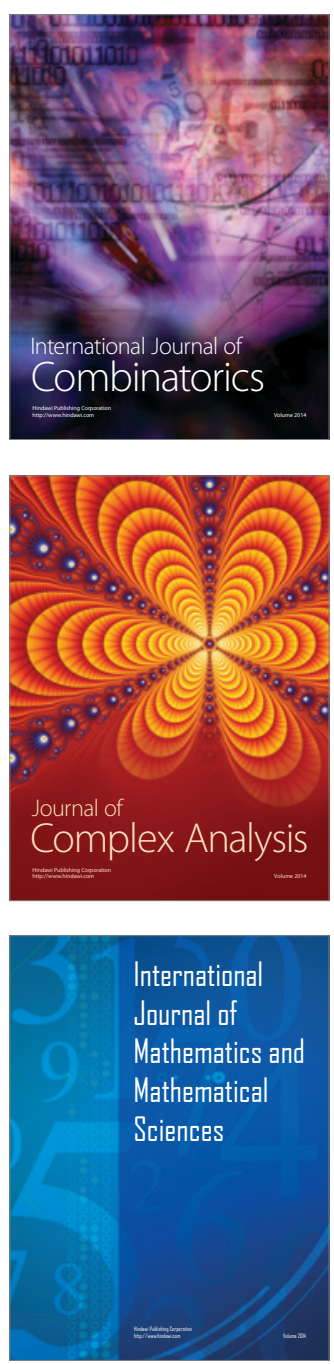
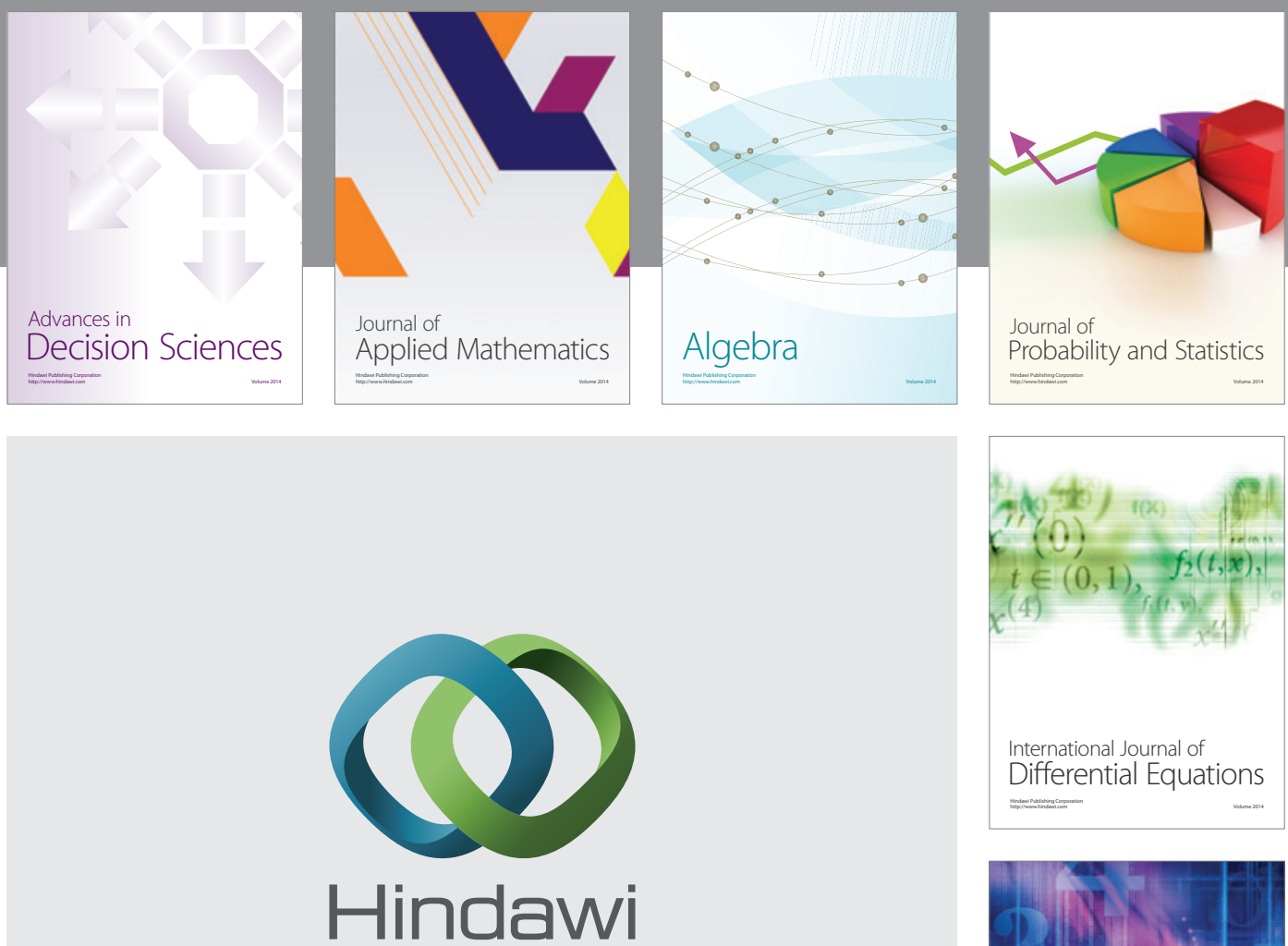

Submit your manuscripts at http://www.hindawi.com
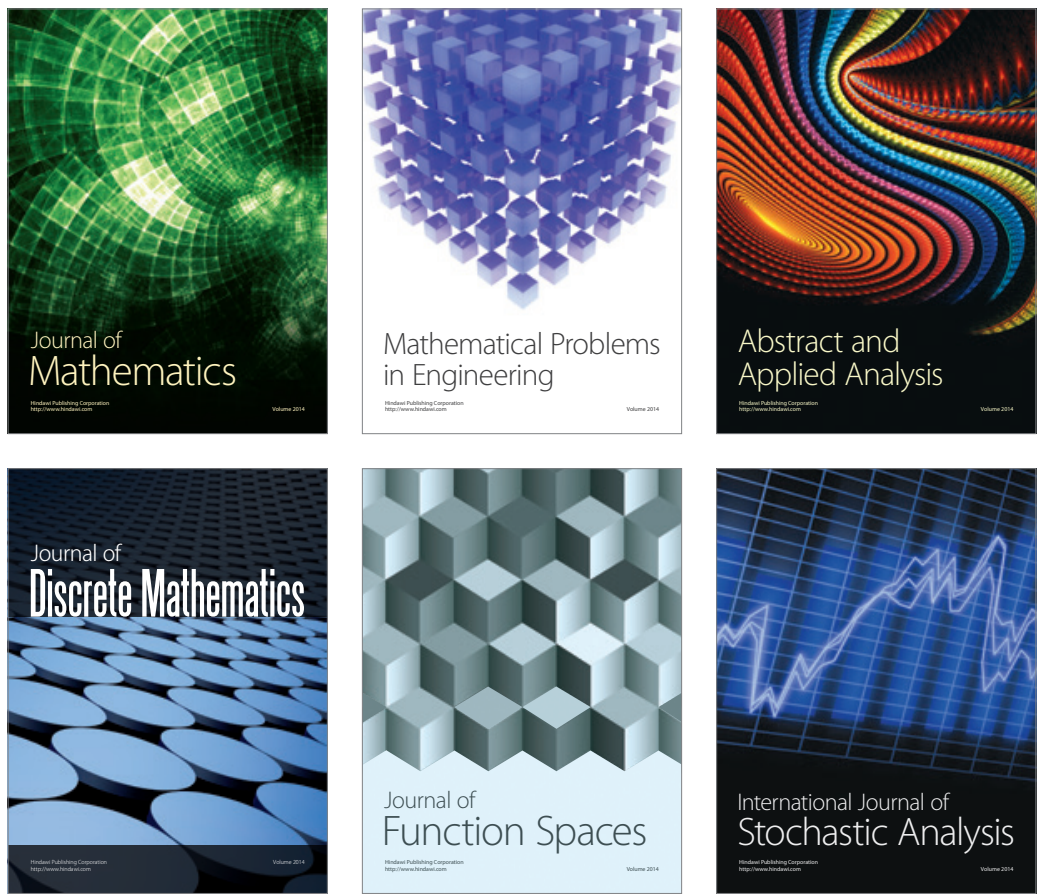

Journal of

Function Spaces

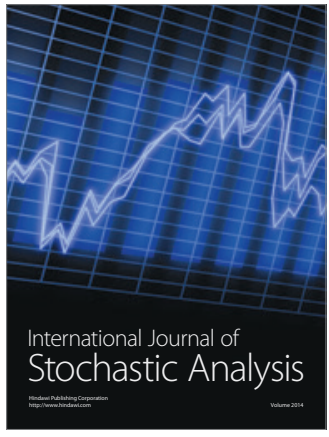

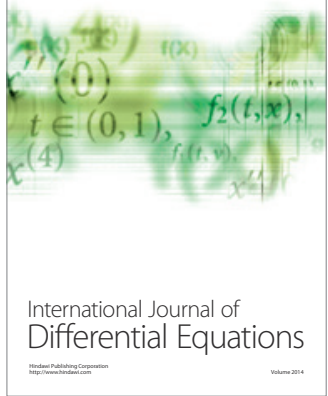
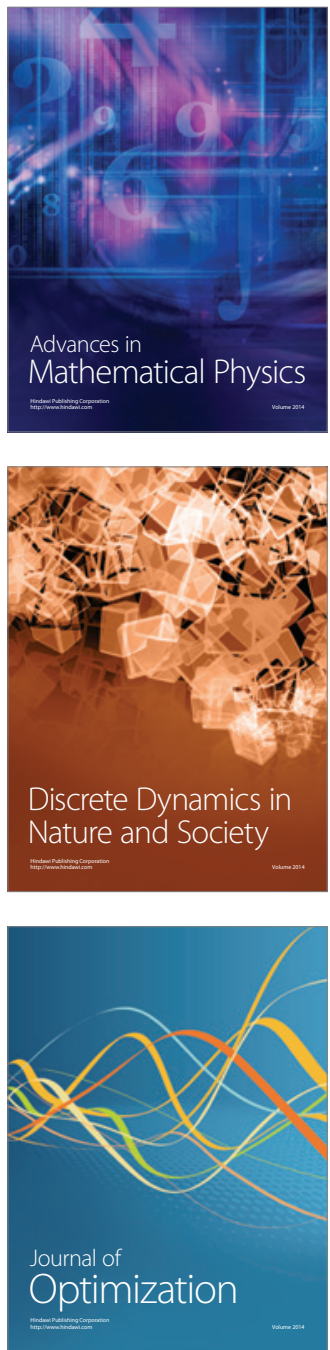